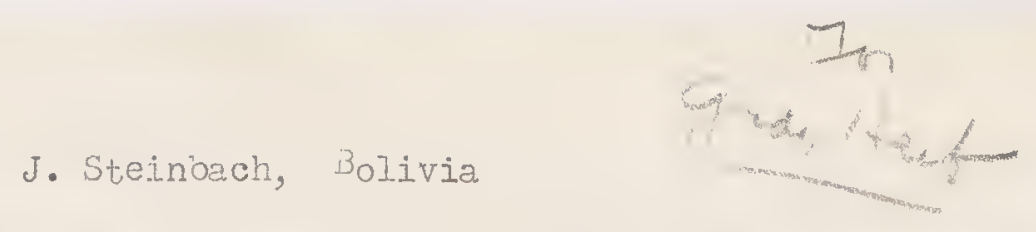

Grasses det. J. ¿. Swallen; others by E. P. Killip unless otherwise noted. Reported by Y.S.I. June 1942

8121. Urvillea macrolopha Radlk.

8124. Pristimera andina Niers (Det. A. C. Smith)

8138. Sisyrinchium tinctorium H.B.K.

8145. Groton beetlei Croizat (Det. Croizat)

8163. Urvillea laevis Radlk.

8341. Randia spinosa (Jacq.) Karst.

8402. Buclileia stachyoides C. \& S.

8457. Euzenia osteomeloides Rusby $r$

8499. Valeriana leptothy ${ }^{\text {Fos }}$ Graebn.

8557. Chuquirąua parviflora (rriseb.) Hieron. (Det. Blake)

8587. :uhlenbeckia sagittaefolia Nessn.

8601. Prunus brittoniana Pusby (vel aff.) -

8625bis. Psoralea lasiostachys Vogel

3663. Cedrela boliviana lusby (type collection of C. steinbachii Harms)

8269. Durantea s erratifolia (Friseb.) Kuntze (Det. Noldenke)

8678. Cheilanthes pruinata Kaulf. (Det. W. 'Paxon)

8715. Tuddleia hypoleuca Kranzl.

8745. Distichlis spicata (I.) Creene

8773. Polyposon Intosus (Poir.) Hitchc.

8789. " " " "

8790. Solanum radicans I. (Det. Jorton) - - -

8796. Chloris virgata Sw.

8797. Polygonum striatum C. Koch. (Det. Erenckle) c -

8799. Thalictrum podocarpum H.B.K.

8809. Jennisetum chilense ( Lesv.) Jacks. *

8828. Arenaria serpens H.B.K.

8872. Prifolium filiforme L. v -

8975. Alchernilla aphanoides tripartita (R. \& P.) Perry

8976. Festuca ulochaeta Steud.w

8981. Geranium fiebrigianum Knuth

8984. Stellaria media. L. w ....

8985. Arenariaxxxy serpens H.B.K.

9030bis. Mriocarpa obscura tiusby

9091. Lasiacis Iigulata Fitchc. \& Chase

3095. Panicum sellowii Nees.

9314. Cajo ohora buraevi Urb. \& Cilg.

9319. Euamoclit coccinea (L.) Noench. (form)

-9468. Poiretia scandens Vent. $\sim-$

9494. Cypems tristachys Boeckl.

9495. Paspalun Iineispatha $\mathrm{Mez}$

9496. Poa annua I. $\mathcal{U}$

9497. Agrostis perennans ("alt.) Tuclerm. v -

9509. Ceranium fiebrigianum Knuth

9530. Gentiana inaequicalyx Gilg

9549. Lolium multiflorum Iam. -

9602. Viola scandens H.B.K. (form) - -

9632. Stina ich (R. \& P.) Kunth v -

9648. Tentiana inaequicalyx Gilg 
Dteinbach -2

9650. Viola banziana Beclcer

9698. Oxalis breviramulosa Rusby $\checkmark$

9714. Setaria argentina Herrm.

9717. Solanum radicans $I$. (det. Horton)

9718. Dicliptera cochabmbensis Iindau (Det. D. C. Leonard)

9783. Calamagrostia tamensis Pilcer $w$

9784. Festuca dolichophylla Presl $\checkmark$

9785. Calamagrostia amoena Pilger

9786. Stipa obtusa (Nees is Mey.) Hitchc.

9789. Calamagrostis antoniana (Griseb.) Steud. $\checkmark$

9821. Tropaeolum infundibularum lisusby

9860. Muhlenbeckia vulcanica (Benti.) Fndl.v

9873. Senecio pampae Lingels. (Det. Creenman)

9895. Centiana bockij Gilg. $\checkmark$

9798. Citharexylum punctatum Greenm. (Det. "olcenke)

\section{Det. Sleumur}

6742. Agonandra granatensis fusby $c$

2346. Heisteria yapamiensis (Kuntze) K. Sch.

7327.

6467. Agonandra granatensis zusby

8599. rockia crucis I.

5818. Abatia boliviana (Iiandon \& Wedd.) Eritton

5197. Sauvagesia erecta $I$.

7585. Ouratea flemosa Fusby

5085. Lacistema myricoides Sw.

8544. Casearia decandra vacq.

5049. Xylosma elegans (Tul.) Tr. \& Planch.

5c58. Casearia aculeata Jacq.

$$
\text { Det. iimer }
$$

8198. Siphocampylus orbignianus A. DC.

5198. Pratia hederacea (Charn.)

8527. Thizocephalum candollej var. vulgare medd.

\section{Det. "oodson}

5475. Landevilla cuspidata (Fusby) Woods.

8573. " superba Herz.

8662bis. " bridgesii (Mell. Arg.) woods.

Det. Lobinson

5229. Tupatorium macrophyllum $L$.

5503. " Ivaefolium $L$.

5380. " microstemon Cass.

Det. Norton

5884. Solanun leptocaulon Van Heurck \& Muell. Arg.

8790. " tripartitum Dunal

9717.

"I 
Steinbach -3

$$
\text { Cestrum (Det. Norton or cited by Francey) }
$$

1346. C. sendtnerianum Mart.

1472. C. calyc "in "illd.

1480. C. reflexum var. densiflomm Erancey

1560. C. baenitzii lingels.

2300. C. sendtnerianum part.

2562. C. calycinum "illd.

3165. C. baenitzii Iingels.

3216. C. reflexum var. densiflorum Fxancey

4004. C. plicatum Francey

512.1. C. sendtnerianum Mart.

5959. C. plicatum Francey $\sim$

6172. C. reflexum var. densiflorum Francey $\checkmark$

6374. C. baenitzii Lingels.

7041. C. sendtnerianum Mart.

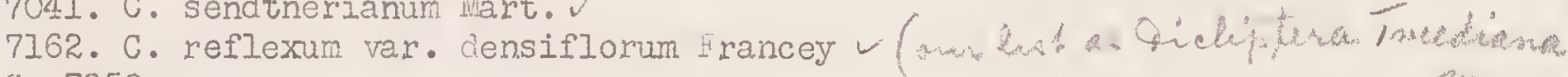
6. $6 \times 2859 \times$

7259. C. racemosum var. bolivianum Francey

9715. C. parqui LiHer.

\section{Calceolaria (Let. Pennell)}

8959. Criaquatica Braun \& Bouché

9775. C. buchtieniana $\mathrm{K}_{\text {ranzl. }}$

9352. C. cryptantha Pusby

8136. . elatior Griseb.

9819. C. herzogiana Kranzl.

8870. C. palustris Dodiro

9764. C. urubambae Kranzl.

5290. Justicia comata (L.) Iam. (Det. Leonard)

5525. Beloperone bangii Rusby

5507. Syngonanthus Gracilis var. bolivianus puhl. (Det. Killio)

5709. Pilea anomala wedd. (Det. Killip)

5757. Valeriana leptothyrsos Graebn. (Det. Killip) 


\section{J. Steinbach, Bolivia}

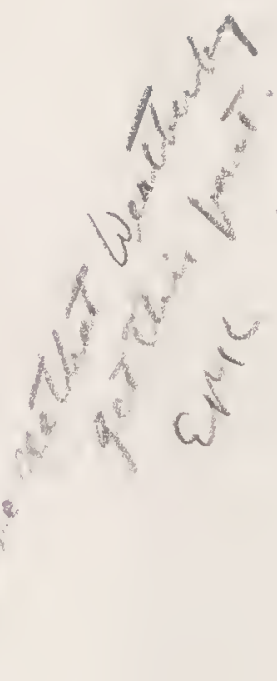

Ferns, det. William I. Maxon, Reported by U. S. National Museum January 1943

7500. Dryopteris sp. Sterile. Not recognized.

8273. Dicranopteris sp. "Sterile and incomplete.

8566. Dryopteris paleacea (Sw.) C. Chr.

8673. Cheilanthes pminata Kaulf.

8734. " myriophylla Desv.

8835. Polypodium aureum $T_{10}-$

8911. Forisetum bogotense H.B.K.

8927. Pteridium arachnoideum (Kaulf.) Maxon $v$

9124. Pteridium arachnoldeum (Kaulf.) Maxon

9272. Asplenium sp. Sterile

9403. Flaphoglossum sp. Sterile and badly broken.

9411. Salpichlaena volubilis (Kaulf.) J. Sm

9487. Equis etum bogotense H.B.K.

7 955K. Polypodium angustifolium Sw. (sens lat.)

9857. Gymnograma elongata H. \& G.

Fhanerogans, det. E. P. Killip unless otherwise noted

5726. Optryosporus steinbachil trobinson (type)

7596. Hexadesmia bollviensis Rolfe

8187. Ophryosporus angustifolius Robinson (type)

8241. Baccharis articulata Pers.

8247. Gochnatia bpliviana Blake $U$

8274. Isochilus linearis (Jacq.) R. BF.

8303. Salvia chorianthos Epling (type) eoltr)

8341. Randia amata (SW.) DC. (R. Rpinosa Karst.)

8386. Tiandia armata (Sw.) DC.

8400. Barnacesia $\sim$

8595. Baccharis dracunculifolia DC. $V$

8604.

8610. " ulicina Hook. \& Arn.

8655. Schkuhria pinnata var. abrotanoides (Roth) Cabrera

8742. Flaveria bidentis (L.) Kuntze

8764. Spilanthes ciliate H.B.K.? (our specimen of foliage only)

8767. Eupatorium patens var. tomentosum Hieron. $U$

8774. Siges heckia cf. orientalis I.

8789. Polypogon interruptus H.B.K. (rather than P. Iutorus; corr. by Chase)

8791. Daccharis glutinosa Pers.

8813. Eleocharis dombeyana Kunth (Det. Svenson)

8875. Salvia oxyphora Briq. (cited by Epling)

8390. Pilea nutans (Poepp.) Wedd.

8972. Zeugites mexicana (Kunth) Irin. (Dot. Chase)

$8983 a$ (Wixed with 8983 ; perhaps part of 9241 [type]) Eupatorium chaparense Gobinson

9165. Cinchona officinalis L. W

924.1. Enpatoriura chaparense Robinson (type)

9450. Calium obovatum H.P.K. (Cited by Standley)

9473 . Borreria suaveolens Mey

9491. Tagetes pusilla H.B.K. 


\section{Steinbach -2}

9561. Fupatorium eonnivens Rusby ?

9577. Gynox 7

3582. Relbunium hypocarpium (L.) Hemsl.

3635. Achyroctine saturioidegs (Cuma .)

3702 bis. Tagetes graveolens L'Her.

9705. Trixis aggregata lausby

9706. Flaveria bidentais ( $\tau_{.}$) Kuntze

9707. Schkuhria pinnata var. octoaristata (DC.) Cabrera

9767. Supatolium

9780. Ganphalium

9790. Seencio pentlandianus DC.

9792. Lupatorium ignoratum Hieron.

9802. Proustia angustifolia var. mollis kintze

0813. Gynoxis

9824. Werneria nubigena H.B.K. (cited by Rockhausen)

3850. Festuca megalura liutt. (Det. Chase)

9873. Senecio parpae Lingelh. (Cited by Greenman)

9876. Senecio floscosus rusby

9878. Mikania cochagambensis Robins. (type)

8839. Mutisia ledifolla Wedd.

$$
\text { Reported by University of California }
$$

8194. Hyptis tafallae Fenth. (Det. Epling)

8701. Conium maculatum L. (Det. Hathias is Constance)

9536. Phacelia magellanica (Ian.) Cov. var. robusta (Brand) Pacbr.

9872.

"

il

II

"

"

(net. Constance 


\section{J. Steinbach, Bolivia}

Det. E. P. Killip unless otherwise noted; January 1944

$\checkmark$ 6039. Tillandsia bandensis Baker (Det. L. B. Smith)

7024. Eichhornia azurea (Sw•) Kunth

7025. " ? (Our specimen without fls.)

7386. Costus steinbachii Toes. (type)

$\checkmark$ 8187. Baccharis = type of Ophyrosporus ansustifolius Rob.

8332. Tibouchina brachyphylla cleason (type)

8379. Peperomia comarapana Trel. (type)

8548. Tillandsia usneoides I.

8708. Juncus dombeyanus J. Gray (var.)

8710. Bleocharis nodulosa (Roth) Uchultes (Let. Bvenson)

8728. Pisoniella arborescens (Lag.l Rodr.\%). 3tándl. (Cited by Standley)

- 8786. Commelina elegans H. ${ }^{2}$.K.

8794. Alternanthera polygonoides (L.) R. Sr.

3813. Eleocharis dombeyana. Kunth (Det. Svenson)

8814. Juncus brunneus Buchen.

8815. Dleocharis dombeyana Iunth (Det. Jvenson)

8857. Piper elongatum (Det. Trelease) 'C. DC,

8877bis. Altêkhanthera lanceolata (Benth.) Schinz

$\checkmark 9030$ bis. Myriocarpa obscura thisby (perhaps already reported)

$\checkmark 9223$. Hedyosmum. Same as æevoral unsby collections reported as

H. glabratum H.3.K.; scarcely that, probably undescribed.

-94.94. Cyperus rivularis var. lagunetto (Steud.) O'Neill (Det. O'Neill)

- 9501 . Colignonia rufopilosa Kuntze (Det. Leonard)

9585. Muehlenbeckia volcanica (Benth.) kndl.

9634. Tinantia erecta (Jacq.) Jchlecht.

9709. Guilleminea denspe (Willd.) Noq.

-9710. Whenopodium incisurn poir.

- 3815. Colignonia rufopilosa Kuntze (Let. Leonard)

s.n. "Prov. Wapara, Dept. Uochabamba, 19-I-1929". Pilea dauciodora (R. \& $\left.P_{0}\right)$ Wedd.

s.n. Radicula nasturtium-aquaticum (L.) Britt. \& Rendle

(Det. Leonard) 
Identification of Steinbech specimens recelved from Academy of Natural Sciences, Philadelphic, Mus. Ho. 122525.

U. 5. National 1.ue exm, March 13, 1932.

- 7664. Solanum (section Lelodendron) (Det. Morton)

- 8121. Urvillee macrolopha. Radik.

- 8124. Hippocratea celastroides H. R. K.

81 8 . Sisyrinchinm tinctorium H. B. K.

- 8163. Urvillea lacvis Redlk.

-831 . Randia spinosa (Jecg.) Rarst.

-8402. Buddlela stachyolies C. \& S.

-8457 . Tugenfa osteomelolies Rusby

- 8490. Veleriana leptothyros Graebn.

-8587 . Mulenbeckia sagittaefolla Moignn.

- 8601. Prunus brittoniana Rusby (vel aff.)

- 8625b13. Psorklea. lesjostachys Vogel

- 866z. Cerrela velloziana Roem.?

- 8678. Chellanthes pruinata Taulf. (Det. T. R. Maxon)

-8715. Bucdlefa hppolouca Tranzl.

- 8790. Solannm reidcans I. (det. lorton)

$-8 \% 97$. Poly gonum avtculere L.

- 8700. Thalictrum podocsrpum H. B. K.

- 8828. Arenarla serpens H. B.K.

- 2872. Trifolium dubinm I.

-8975. Alchemilla ephan ofies tripartite (R. \& P.) Perry

-8981. Geran 1um ffebrl gianum Knuth

- 8984. Stellarta media I.

- 8985. Arenaria serpens E. B. K.

- 9030b1s. Myriocarpa obscura Rusby

- S\&I4. Cajophora buraev1 Urb. \& G1lg.

- 9310. Quanoclit coccinea (L.) Moench. (form)

- 9468. Poiretia scandens Vent.

09494. Cyperus tristachys Poeckl.

- QE09. Geranium fiebrigianum Knvth

-9602. Viole 3candens E. B. K. (form)

-9650. Visla bandena Becker

-9681. Verbena bonartensis I.

09698. Oxal1s breviramulosa Rusby

- 9717. Solanim racicans I. (det. Morton)

-9718. Licliptera cochabambens is Indau (Det. E. C. Leonard)

- 982.1. Tropacolum infundibularum Rusby

-9860. Mrhlenbeckia vulcanica (Fenth.) Endl.

- 9895. Gentiana bock11 Gilg.

-8203. Escallonia paniculata var. acuminatissimum Kuntre 
Bolivian Compositae-Eunatoriea coll. José Steinbach. Determined by B. I. Robinson, April 1929.

8151 Tupatorium schlokendantzil Hieron:

8187 Cphryosporus angustifolius Roblnson, n. sp.

8193 supatorium aidymum ratt

$8519\left\{\begin{array}{l}\text { Mikania punctata Klatt } \\ \text { Senecio sp. }\end{array}\right.$

8545 Inikanie camarapensis Robinson, n. sp.

8619 Bupatorium patens, var. tomentosum Hieron.

8767

$"$

" $"$

I. telubech

\section{0}

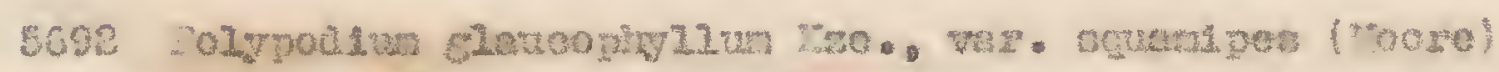

Steinbach $\sqrt{ } 5085$ Jacistema myricoides Sw.

8544 Casearia decandra Jacq.

$\checkmark 5049$ Xylosina elegans (Pul.) mr. \& PI.

15058 Casearia aculeata Jacq.

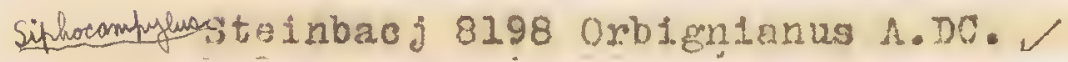

Steinbach 5198 pratia hederacea (Cham.)

8527 Phizoce haluru Candollei Wedd. var, vulgare wedd. 
- 5884. Ieptocaplon Van Heurek \& M1111. ArE.

$\times$ 8790. tripartitra Dunas

9717. tripertitum prasel (not 8. Kadicens) 
itoinbach

5856 Gaultherie rufescens DC.

7195 Solanum iandonts van Heurck " Mell, MrE.

8283

8432

8525

8526

8176

8624

8636

8610

8280

8637

8711

8725

S1da rhombifolia I.

Solarium nigrum $I$.

" leptocarion van Heurek \& Muelil. Mre*

Terminalia australis Carb.

Solanum radicans $I$. I.

slsymbriliolium Ian.

Baccharis ullcina $\#$ "E. A.

Osteomales forruginea HBK.

ifandevilie erecta (Voll.) Voodson

jola nun ljo101des I.

8790 radloans L. I.

9132 poperonta hiepldula (Sw.) A. Dietr.

9713

9717

bolanum sisymbrifolium Iam.

" radicans I. 1 . 


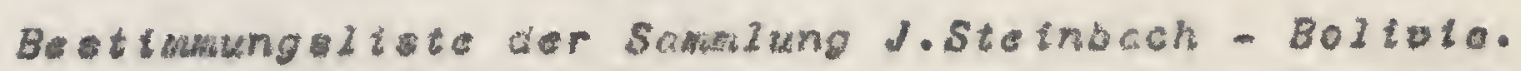

No. 904 Solance. Solanum alo daw Dun.

922 Scrophul.Bacopa Salanonnt (Bth.) Chod. et Hesol.

931 Lythrac. Cuphea spoctosa (Ancere) O. Ktse.

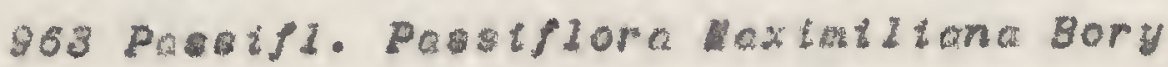

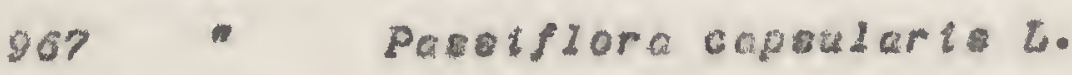

971 Violac. Bybanthu comunte $(S t .-H t 1$.$) raub.$

104l Gutitif. Vianta coyennensts Pers.

1006 Grom. Homolepts atufenels (Xunth) Choce

1099 Selogtn. Selogtnel2a sulccta (Desu.) Spring

1215 solanac. Solonum unbe $11 \mathrm{a}$ tum 111. var. pont sect $\operatorname{set}$ Bt.

1120 Labigt. Marsupianthe chawedrys O. Kat.

1.30 Come 1. Dichortochera Aubletiana R. I Sch.

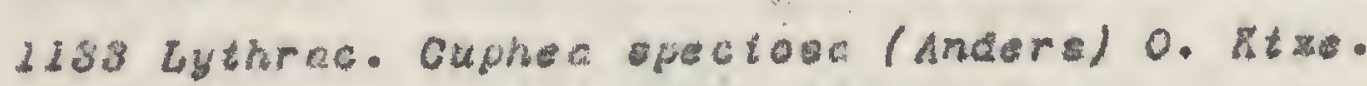

1152 Lablat. Hyptio Lantancefolto Pott.

124s Borrag. Hel lotroptua titridtoldes Chem.

$1426 \mathrm{Selag} \mathrm{in.}$ Selagind lie Bangth Hieron.

1495a Comine 1. Commel tna nud iflora $L$.

1565 Laurac. Phoebs trtplintrvis (R. ¿ P.) tes

1800 Lablat. Scutellarta purpurascens St.

1023 Guttif. Vtsmia dayennensis Pera.

1901 Cyperca. Rhynchosporo setacea (Borg.) Boeck.

1921 solande. Lyctonthe atartfolta (Kth. et Bcht.) Bitt.

204 Solanac. Capelcun boccotum $L$.

2048 Borrag. Heliotroptun tiariatoldes Cham.

2080 comul. Tracescantia gentculata Jacg.

2050 comine 1. Cominelina nud lfioro $L$.

2058 selapin. Selaginalla Bangit Hieron.

2179 solance. Solanub gronulost - Leprocum oun.

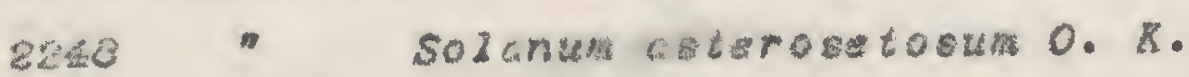

geo6 Lythrac. Cuphe opecsoen (Andere) O. Btac.

2328 solonad. Brachtstus tetrandrus (A.Br.t Bchs.) Benth. 
Ho. 2628 Conmel. Athyrocarpu particartasfoltus C.B. Clark

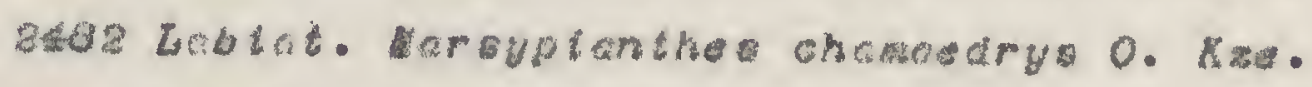

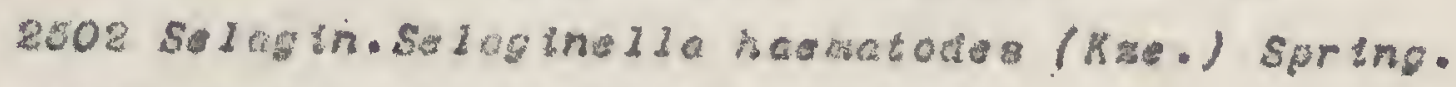

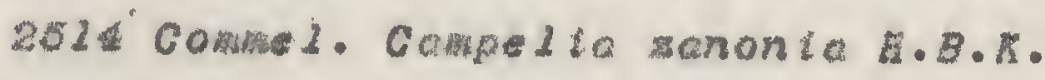
2025 Solande.Capaicum baccatum $L$. 2039 Labiat. Hyptis odorato gth. 2700 Solanac. Solonula albiak Dun. 2719 Lablat. Scutallarta rustcifolta Kth. 2747 Comul. 1. Tratisacanta gentculata Jeco. 3795 Bignon. Amph 120 phtuk fontculatum B.B.X. 2072 laljtgh.Galphtwa brasiltangt (L.) Juas. 2090 Solance. Lyelanthes sec. 3040 Phutol. Rivina humitio 6 . 3173 Btanon. Lundie spruce ana Bur. $328 \%$ Conmel. Comwe lina apec. nim. incompleta

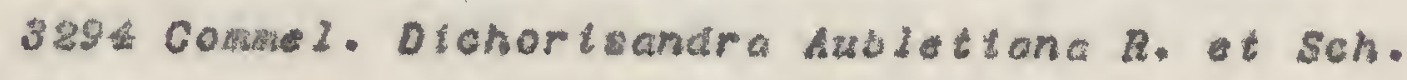
3ezl Cup. Sclerte lithoeperna Willa. 3475 solanac. Lyetanches apec. s730 Thane. Eurya zungartae (FuL.) Sayes. 37to selcoin. Selaginelia radiate (Awbl.) Bek. $3702 \mathrm{scroph}$. Gerarata lanceolata R. et P. s78s Anccora.seninu dependens ort. vor. Gnatnus snpl. 3097 Labiat. Wicromerla Gliliekit Bth. 3906 Solanac.solanum abut110iaes (Grigob.) Bitt. et Lt110. var. htbiecifoliun (RuBb) Bitt.

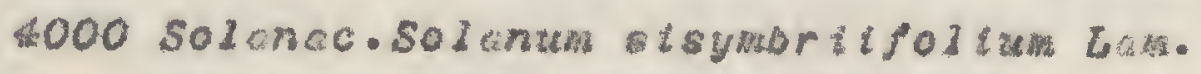

booav Lythrac.Cupheo fonthine Koehne

soso srpina. Watsyb ste inbechti helch.

cooz scroph. Scoporia dulots $L$.

sool Gat if. Vhamb cayennenst pera.

boos Supind. Sarjanta spe.

5107 Lnotat. Iyptio 2 anteneefolke Pott.

5213 "Wyptis hirsute Rth.

0125 Plutol. Hivina hund1Ls L. 
No. S254 Leg. Diaboganax Swartatajorort Harme

5150 Luthrie. Adenarta floribunde R. B. K.

0179 Lablet. Hupt to Acrophylla Pond

5191 Scroph. Buechnero elongato St.

5199 scroph. Linaernic atffuga (L.) Hettot.

t200 Scroph. Eecobedte Gcabrifolla R. et P.

G224 Commel. Dichorigandra v\$1206ula tart.

5225 Pastifl. Paseiflora foetida $L$.

s240 Lythrac.Cuphec epectosa (Ancera) O. Rtse.

5248 Bignon. Arrabldea flortá $D C$.

525e "Petastona camoted tere

$\checkmark 253$ Thynnenthus laxtflorse biers

520s Labiat. Hyptia breotpes Pozt.

ba7? solan. Solanu umbellatum v11.

war. prontactis Bitt.

5292 Conme 1. Commating nuelfioro L.

5327 "Cowmelina wirgintca $L$.

s.57s solan. Capetcum baceatsh $L$.

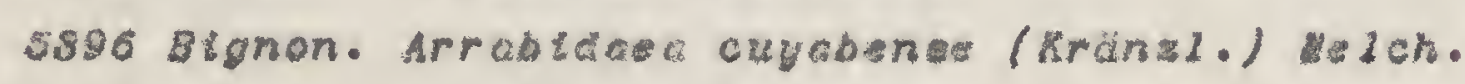

rsou Scroph. Scoparte dulcts L.

sw20 Scroph. Becopa Salmanntl (Bth.) Chod. Et Hasol.

5514 Labtat. Hyptio aucveolens Polt.

5380 solan. Brachtstus tetrantrue (A.Br.et Bcht.) Benth. ot

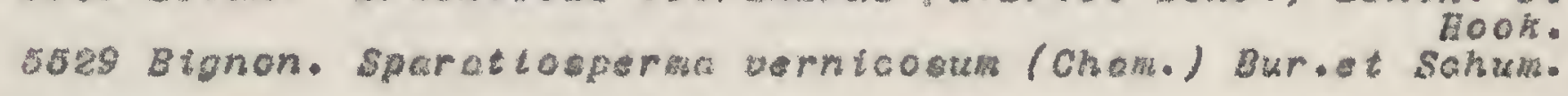

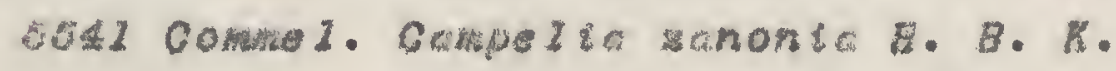

sose Comme 1. Praciscant to gentculato Jnog.

5550 solan. Capsicum Daccetur L.

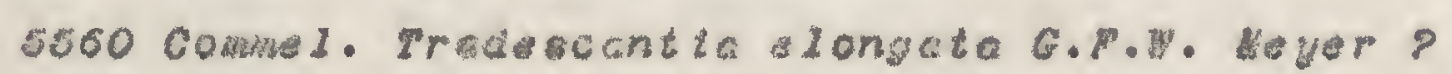

otpo Campan. Centropogon cornutus (L.) Druce

(C. eur inawensis (Z.) presi)

sos? Sapind. Sarjanta spac.

5600 Lythrac. Anmennto curticklata willa.

6607 Aral. Didunopanas worototont D. et Pl. 
Mo. 5756 Cunon. We inmannia glabra Z. B. K.

0045 Lablat. licromeria Githesti Bth.

5908 Ance. Serinopeto spec.

5027 Cunon. We tnmannia Buchtenti Bnol.

5962 PIumbag. PIunbego coorulea E. B. X.

6990 scroph. Gerardio Lanowolata R. e P.

6017 solun. Lyeitontho lyctordss (L.) Hoss.

oubspec. parvifolic (nede.) Bitt.

6039 Brome2. T112andata bendensts Bek.

6042 solan. Bassoola spina alba Griseb.

0135 cyp. Cyporue atffusus Vahl subsp. chalaranthue Prost ver. umbroous (Lind?. Nebs) Kin.

- 6110 Cup. Hadoocharts mintwo Kunth

uar. plurtflora Ritknthel

6159 Labiat. Uarsyptanthe chastearys O. Kae.

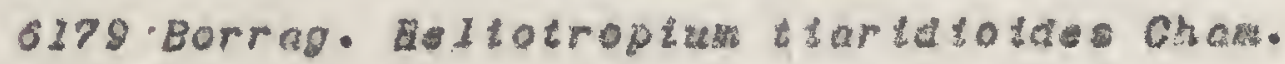

0106 Lablat. Eyptis I antanasolta Pott.

- 6107" Buplse reourvato Polt.

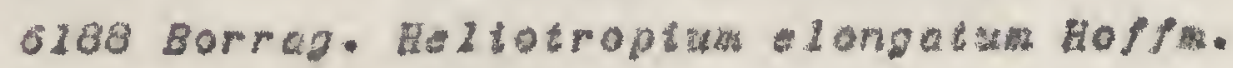

6262 solan. Solantm pranulost-leprosum Dun.

6279 Eorrog. Raltotroptuth tartatotus Chan.

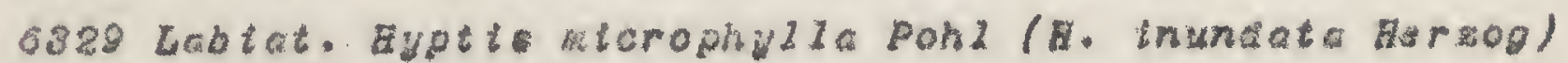

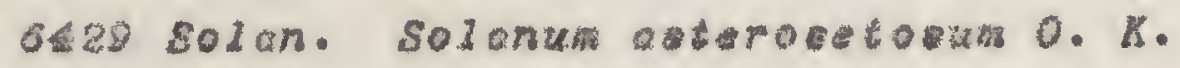

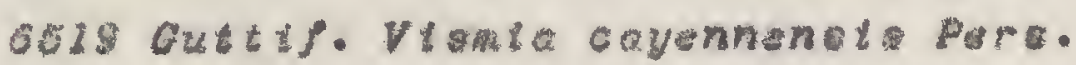

ssse Euphorb. Acalyphe alveretjolta

6623 Valptoh.Gelphtmia braetlianste (L.) Juat.

6674 Cyp. Fhynchosporo corymbosa (L.) Britton

6789 Labtat. Scutellarta purpurazens Su.

6010 Selcoin. Selcotnella rubescens Hiton.

6016 suphorb.Acaluphe tanoloba M.Arg.

- 6035 Grath. PCspolun destichun L.

6010 Gran. Pantcum polveonotula schrot.

6056 cyp. Cyperus flouks (Vuhl) Nees

var. paduncularta Brition.

0860 Cyp. Sclerta pterota Presl. 
No. 0076 cuperac. Cypars infucatue Runth

orr. poucinervosus Xikenthod, or. nova.

6983 Gran. Paspalum Skmensonum Henraro

6942 Lablat. Euptis hireuta Rth.

7013 Graw. Homolepis aturonsts (Kunth) Chose

7017 Lahtat. Ayptis lantencefolia Pott.

xpos Cup. Rhynchospora Gura Vohl.

(B. corymbose (L.) Brition.)

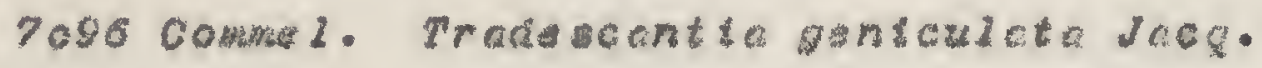

7130 Borrag. Heltotroptum $=1$ ongatun Eoffa.

7167 solan. Solonwh granulo $1-1$ sprooum Dun.

$7172 b i s$ Laum. Phoebe triplinervis (R. \& P.) Ma

7179010 " " " "

7194 Solan. Lyotanthas pamalyctotae (Chod.et Easel.)

$7190 \mathrm{sol \textrm {kn }}$. Solonuth alblam Dun.

Bit ter

$7202 b i s$ Gutt. Vtamia cavennanste Pers.

7203 Lablat. Scutsilarto purpurascens su.

7319 Comme 2. Comm 2 inc nudtfora $L$.

7363 solan. Solonua albidum Dun.

7370 Walpigh. Galphtwta brastitensis (L.) Juss.

7 \&65 Ponteder. Eateranthera 1 tmosa Vahl

7475 Warant. Saranthe urodolate Peter.

$7 \leq 77$ Husac. Heltconta eptecopaliv Veli.

7189 tablat. Scutallarta furpurascens sw.

$7602 \mathrm{Selagin.} \mathrm{Selaginella} \mathrm{oncens} \mathrm{Pros} 1$

7560 Narant. Wonotogma plurteptcetum (Roorn.) K. Schum.

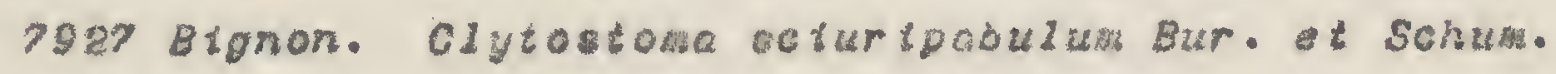

volg Protel. Paseiflora Warminglt Uaet.

vol "Pass

b30 scroph. Calcealarta sec.

81to Dhytol. Rivina numblis L.

01 sis Vtoloc. Hybanthus parvifloru (Bent.) Basli.

o191 tuthrac. Cuphea spec. 


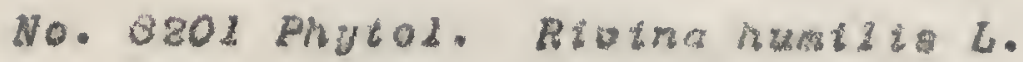

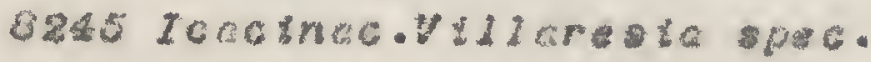

best Cunon. Vetriannia sorblfolla H. B. K.

3309 Bignon. Tecomt cochadumants (Herzog) Velch.

QSed Vtolac. Vtola pyomed Poir.

0870 Luthrac. Cupheo spec.

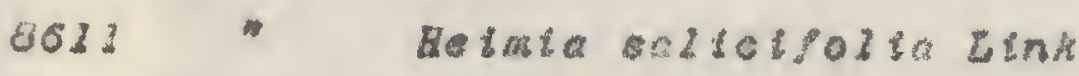

0012 Bignon. Tecoma cochebambenole (bersog) Welch.

6023 scroph. Gerardito lenceolota $R$. et $p$.

8638 Paseifl. Preconta unb11teata Griseb. (planta pulcherrtmal

S686 Elsocorp. Vallea stipularts L. P.

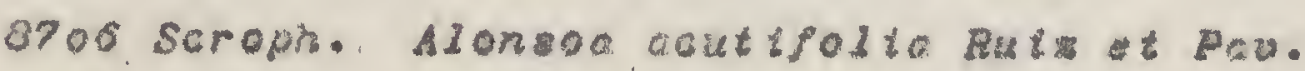

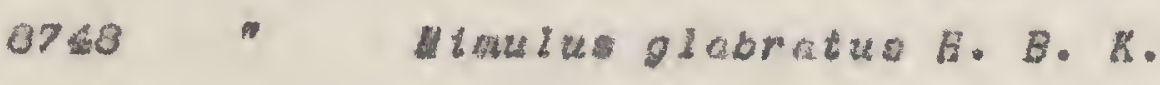

aros "Gerardta lancelate $R$. t $P$.

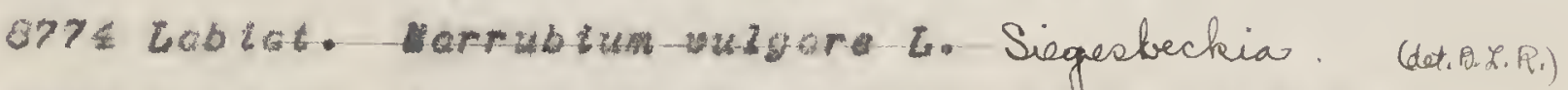

0778 Bipnon. recoma cochababanets (Gersog) welch.

Bost Conp. Stavin obougta Rugby. Oer. artatifariz Babinson, var. now.

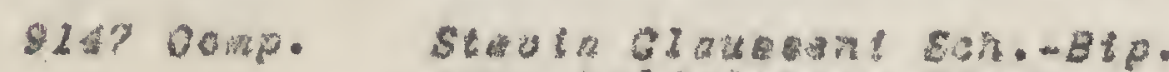
or. boltuienata ficron.

9241 Comp. Bupctortum chaparense Robinaon

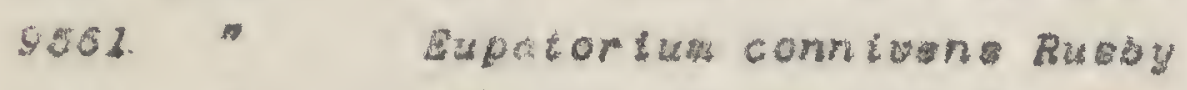

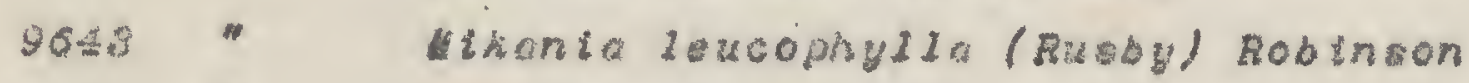

9600 "Ageratum conviotides $L$.

vor. Incequetpleden bieron.

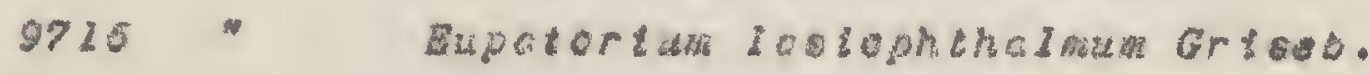

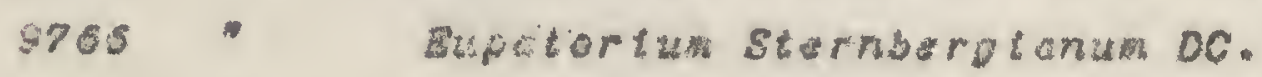

9767 " Ophryosporua Cuwingt (sch.-Btp.) Benth.tBook.f.

s292 Eupatorium cangurosno sch. $-38 p$.

9021 " Ophrvobgarue Cingingt (sch.-Blp.) Benth.diook.f.

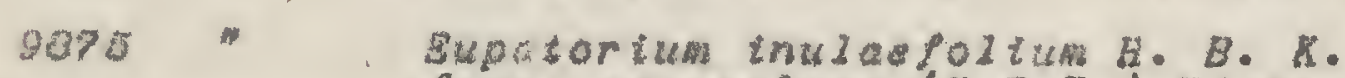

forma suabolans (B.B.R.) Bieron.

go7e "Whank cochbambano Robineor n. sp.

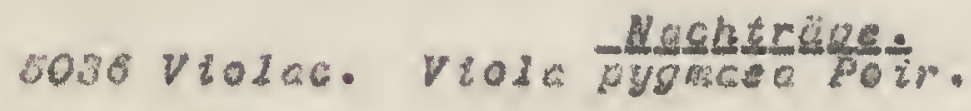

seg7 Lobiat. Octmun micranthum ilid. 
No. 6085 Comp. Baccharis

6096 Euphorb.Croton

6096bis" Croton

6116 "Crotonglandulosus L. var. scordioides M.Arg.

6160 Polygal.Polygala spec.

6169 Solan. Sol anum

6190 " Physalis

6216 Caryoph.Drymaria

6261 Solan. Physalis curassavica L. vel aff.

6263 Logan. Buddleia spec:

6269 Caryoph.Drymaria

6277 Solan. Nicotiana

6278 "Nicotiana

6287 "Solanum

6291 "Solanum

6314 Laurac. Ociotea Iaxiflora (Meissn.) Hez

6320 Solan. Physalis

6359 Laurac. Nectandra truxillensis Mez

$637 \leq$ Solan. Cestrum

6446 Iridac. Sphenostigma spec.

6457 Leg. Steinbachiella leptoclada Harms n. gen.

6182 "Ohne Standortszettl"

6510 Euphorb.Phyllanthus

6542." Phylianthus

6569 Iridac. Eleutherine plicata Herb. ex Griseb.

6583 Solan. Solanum

6587 Amarant. Hypoxis

6610 Borrag. Heliotropium Iagoense Gürke

6623 Laurac. Nectandra Iaevis Mez

6627 Solan. Solanum

6663 Laurac. Persea cordata (VeII.) Mez

6676a Apoc. Dipladenia velutina (Stadlm.) K.Sch. var. glabra M.Arg.

6705 Filic. Ceropteris calomelanos (L.) Underw. 
No. $6730^{\circ}$ Logan. Strychnos Hartii Prog.

6775 Laurac. Ocotea llerowyneno:s liez

$6789 "$ Persec coráci (Vell.) Wez

6804 Cyperac.Bulbostylis copilleris Kunth

ver. tenuifolta (Rudge) C.B.Cl.

0912 solan. Physalis ofr. viscosa L.

6964 Iridac. Cipura paludosa Aubl.

6985 Laurac. Endlicheria chomala Nees

6999 Euphorb.Croton glandulosus L. var. scorditoies H.Arg.

7115 Uusac. Eieliconia

7123 Sapind. Cardiospermun (?) spec.

7167 Solan. Solanum

7201 Polygal. Securidaca volubilis L.

7208 Hippocr. Salacia

7221 Aguifol. Ilex

$7227 b i s$ DiIl. Curatella cmericana $L$.

7229 Dillen. Tetracera

$7233 a$ Gran. Guadua

7251 Euphorb. Phylianthus

$7251 b i s "$ Phyllanthus

7258 Laurac. Ocotea Warowynensis Hez

7259 Solan. Cestrum

"200 Laurac. "Lauracec", nicht näher bestimmbar!

7204 "Enalicheria anomala Nees

$7263 b$ is Morac.Cecropia leucocoma Viq.

7271 flac. Casearia

7231 " Casearia

7233 Lab. Scutellarta

7293 Celastr. Maytenus

7294 Hippocr. Hippocratea

7299 "Hippocratea

$7298 \mathrm{Leg} \cdot \quad$ Desmodiutic

7305 Polygal. Securidaca spec. 
No. 731s Rubicic. Coussarea contracta ll. Arg.

$7315 b$ ts Euph. Phyllinttise

7316 Dilien. Curctolla awericena $L$.

7313 Hippocr. Eippocroteo.

7320 Verban. Lippia

7323 Euph. Acalupha

7321 Flac. Xylosma

7326 "Casearia

7320 Grall. Andropogon

7332 Borr. Corcia

7337 Caric. Carica gossyplifolia Griseb. var. glabrata Herzog $73 \pm 5 \mathrm{Flac}$ Casearia

7318 solan. Solanum

$73480 "$ Solanum

7350 "Physalis curassavica L. vel aff.

7359 Bignon. Arrabiacea spac.

7379 solan. Solanuin

7333 flac. Caseariza

7030 Zingtb. Costus steinbachit Loes. n. sp.

7339 llarant. Calatho

7591 Laurac. Ocotea Warownensis Wea

7 sis Vurant. Calatiea

7598 Rosac. Prunus

$7 \leq 03$ Pilic. Dryopteris serrata (cav.) c.chr.

7408 Cannac. Canna

7417 Leg. Bauhinia

$7 \leq 27$ Husac. Heliconta

$7 \leq 01 "$ Helicionia

$7 \leq 38 "$ Heliconta

7142 Scitam. Renealmia spicata Gagnep. vor. vel aff.

74 L Laurac. Persea coraata (VeII.) Vlez

740 Borrag. Coraia

7455 Lentioul. Utricuzaria obtusc swortz 
No. 7458 Dioscor. Dioscorea cubijensts Knuth.

7460 Ehawnac. Sageretia elegans Brongn.

7465 Ponteder. Eichhornia

$7 \leqslant 67$ Solan. Solanum

$747 \leq F i l i c$. Gleichenta flexuosa (Schrad.) llett.

7478 Musac. Helicoria

7484 Uarant. Calathea

7.485 sapind. TaIisia?

$7493 \mathrm{Leg}$. Desmodium

7499. Filic. Polybotrya caudata Kunze.

7501 . Asplentum cf. semicoraatum Radai

7502 "Stenochlaena erythrodes (Kunas) underw.

$750 \leqslant$ Lab. Salvia

7511 Wusac. Heliconia

7013 Dioscor. P Dioscorea coriacea H. et B.

7520 Bignon. Godmania Ubana Kränzl.

7522 Musac. Heliconta

7524 Violac. Hybanthus communts (St.Hil.) Taub.

$7525 \mathrm{Leg}$. Hachaerium angustifolium $K m e I$

7562 selogin. Selaginella

7565 pilic. Lepicystis ciliata (Willd.) Retm.

7570 Hippocr. Salncta

7571 Rublac. Cephalis tomentosa Willd.

7578 Euphorb. Uabea longifolia P. et R.

7581 Husac. Heliconta

07534 Comip. Wikania

7586 Theophr. Clavija aff. Serratae (Hoffngg.) Wez

7588 Solan. Solanum

7589 filic. Eschatogramile furcata (L.) C.chr.

$7590 " V i t \operatorname{tar} i a$

$7592 "$ Trichomanes

7605 Eignon. Wacfadyena uncinata $D C$.

7611 Rubicc. Ceproelis

7616 orchid. Epiaenarum fragrans Sw. 
No. 7619 Fungi Physarumbogoriense Ractb. (Ph. pallidym Lister)

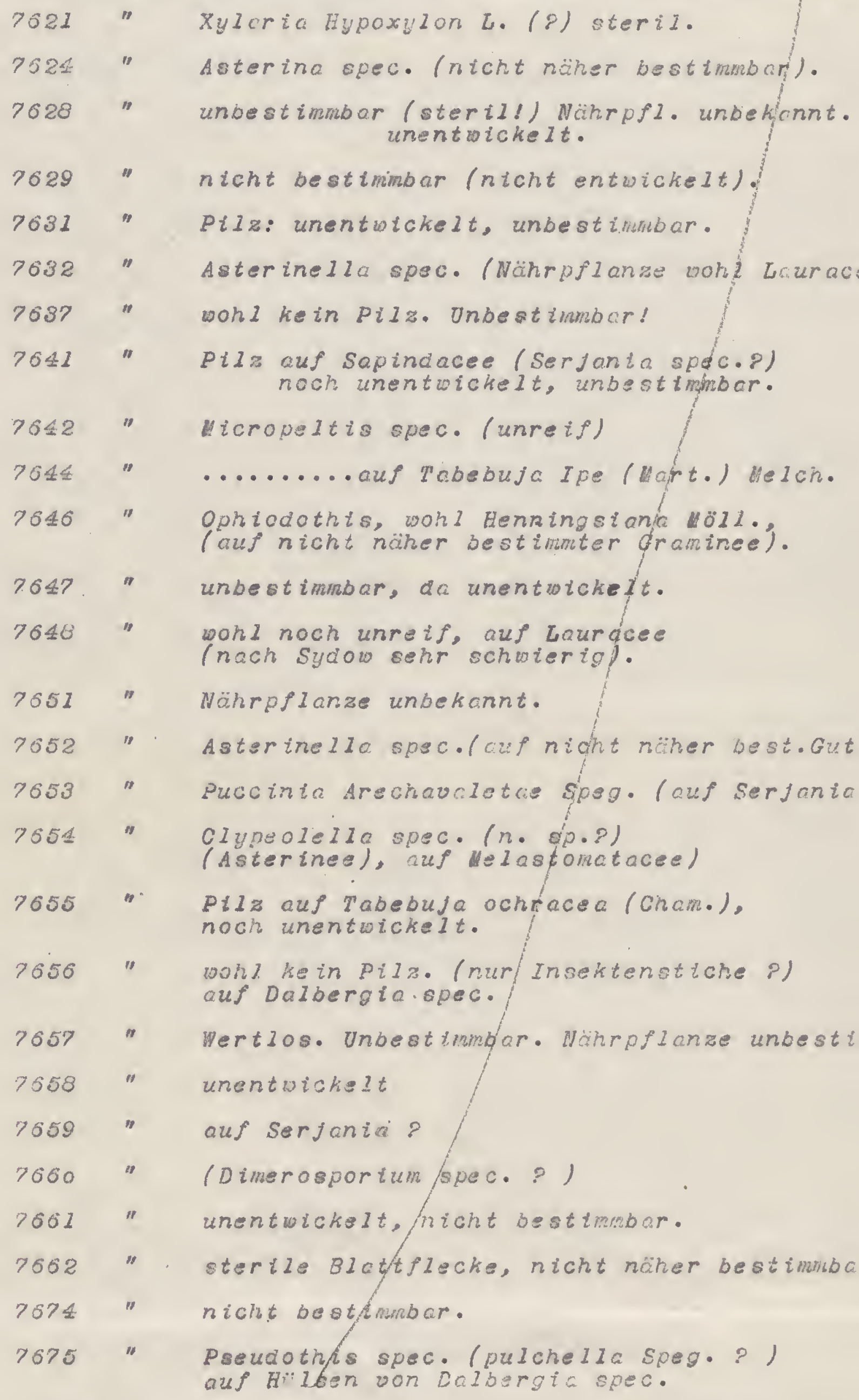


No. 7776 Fungi Helminthosporium Ravenelit Curt. et Berk. (auf sporobolus indicus (L.) Br.)

\begin{tabular}{|c|c|c|}
\hline 7781 & $"$ & unentwickelt, nicht best \\
\hline 7782 & $"$ & $\begin{array}{l}\text { Aecidium spec., da Nahrpf } \\
\text { kannt, (viel1. Piper), ni }\end{array}$ \\
\hline 7783 & $"$ & 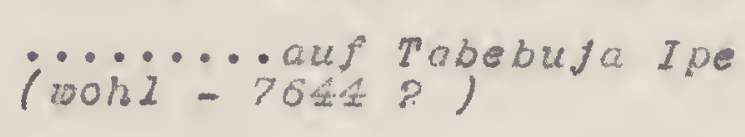 \\
\hline 7823 & Orch. & Scaphyglottis \\
\hline 7821 & " & Hormidtum tripterum Cogn. \\
\hline 7825 & $"$ & Lantum \\
\hline 7826 & $"$ & Pleurothalitis \\
\hline 7827 & Dios & Dioscore \\
\hline
\end{tabular}

78650 is Orch. Trigonidiun

7881 frungi Kylaria spec. (eugiossa Fr. P)

7905 Rubiac. Calycophyllum Spruceanum Benth. t Fook.

7915 pilic. Leptcysts voccinifolin (L. et F.) Diels

7917 horac. Sorocea pseuciosaxicola hilabr.

7918 Celastr.Haytenus

7920 Erythr. Erythroxylon

7922 Wyrt. Evgento

7925 llest. Wiconta

7926 Rubioc. Uruparia guianensis AubI.

7927 Bignon. C1ytostoma

7920 Leg. Lonchocarpus

7929 Diosc. Dioscorea

7930 Rubiac. Farane a

7936 thelast. Leanara

7937 Leg. Piptadenia

7938 thelast. Wiconia

7939 Gram. Andropogon

7940 Hayac. Haykea

7941 Fungt Pilz auf Contretaces (Terminolin od. Buchenaric) (seirr schwierig au bestimmen, viell.neue Gattung).

$7916 \quad "$

Ustilazinotiea ochracea P. Eenn. (auf setaria sulcota ( ) Racial.) 
Wo. 7954 fungt Phyllcichora lwachaericola (P.Henn.) Thetss.et Sya. (uuf Wachaerium opec.)

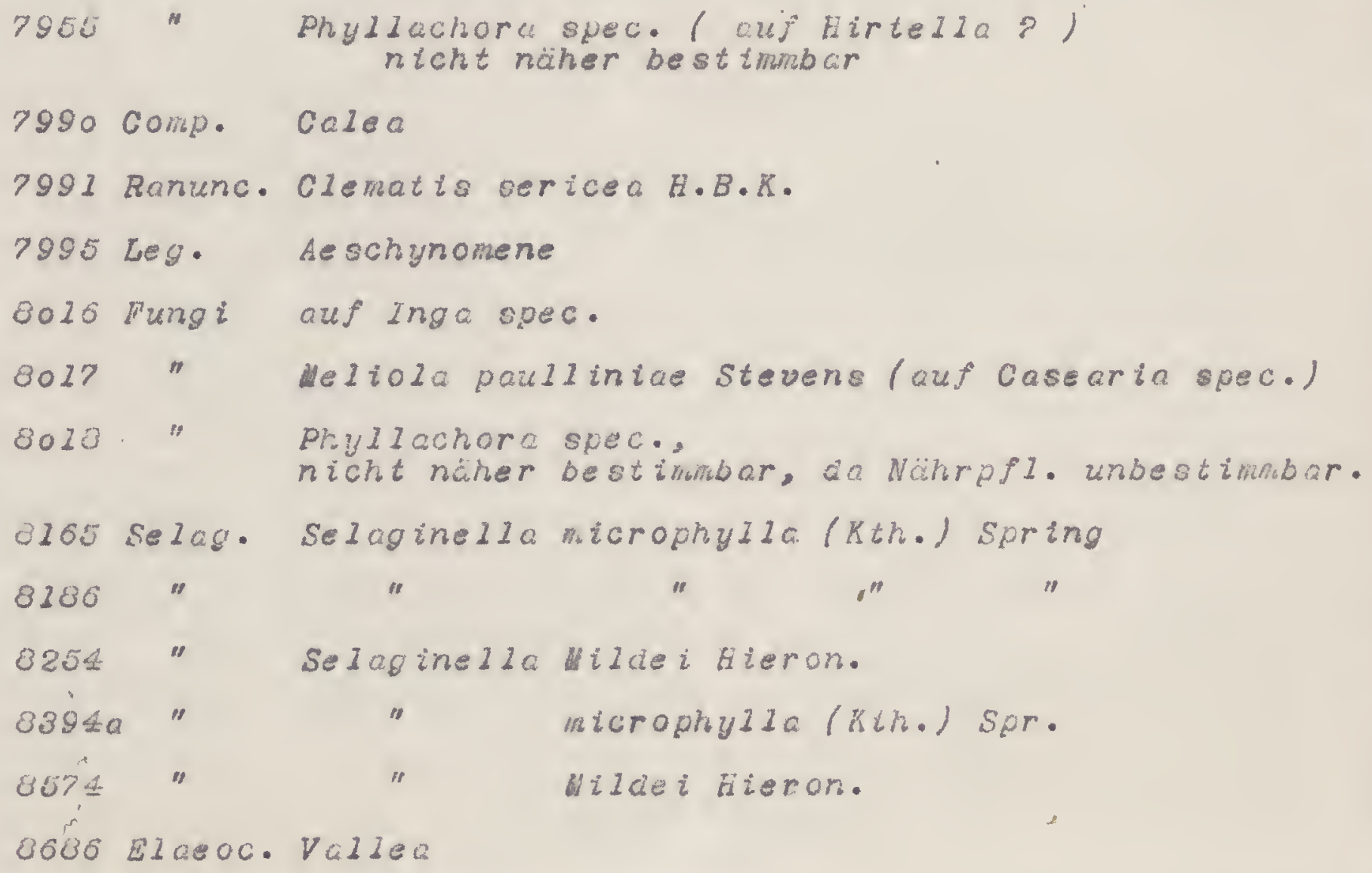


Bestimmungsliste J.Steinbach-Bolivia.

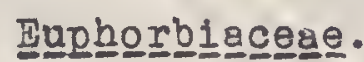

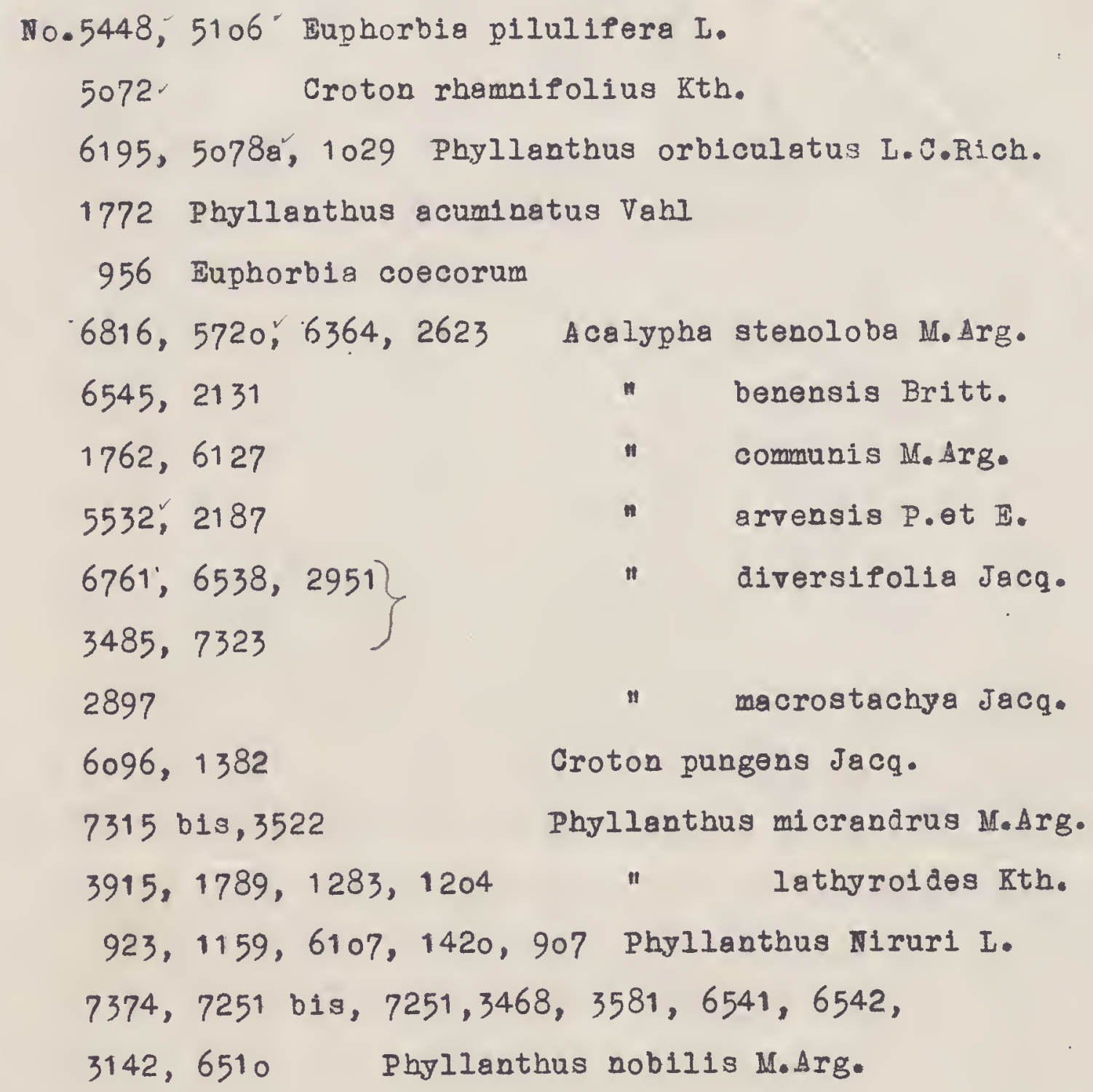

